\title{
EXAMINATION OF THE FASSHAUER'S ALGORITHM USABILITY FOR SOLVING UNSTEADY HEAT FLOW IN A MEDIUM WITH SPATIALLY VARIABLE THERMAL PARAMETERS USING THE KANSA METHOD
}

\author{
Popczyk O. , Dziatkiewicz G. ${ }^{* *}$
}

\begin{abstract}
Algorithm proposed by Fasshauer and Zhang is by today one of the most popular and the best established algorithms for finding a good shape parameter value for the Kansa method. However, it turns out that it is not great for all classes of numerical problems, for example for the problem of one-dimensional unsteady heat flow in a medium with spatially variable thermal parameters. In this work, the Fasshauer algorithm was investigated for solving such a problem using pseudospectral formulation of the Kansa method. The work also proposed a modification of this algorithm that makes it work better for this class of problems. The modification is that instead of looking for the minimum cost function, one should look for an argument in the cost function graph for which the oscillatory behavior begins.
\end{abstract}

Keywords: Kansa method, Multiquadric radial basis function, Heat equation.

\section{Introduction}

Heat equation is one of the most fundamental parabolic partial differential equations which causes that it is often solved in both the world of science and industry. However, it is usually solved with the assumption of spatially constant thermal parameters, this is probably related to the fact that materials with spatially variable thermal parameters do not occur in nature quite often. Nevertheless, in recent decades, thermal metamaterials, which may exhibit spatial variability of thermal parameters have gained considerable popularity in the scientific community - Vemuri et al. (2014), Tschikin et al. (2015), Huang et al. (2018). Due to this, modeling and solving problems involving heat flow in materials with spatially variable thermal parameters is becoming an important issue in computational mechanics. The problem of one-dimensional unsteady heat flow in a medium with spatially variable thermal parameters is considered in the paper. In order to solve partial differential equations (PDE) describing the problem, the pseudospectral Kansa (PSK) method was used. Kansa method is meshless method which utilizes the radial basis functions (RBF) for solving PDEs. It was introduced in 1990 by Kansa (Kansa, 1990) and named after the author Kansa method. Since then, Kansa method has been used in a wide range of problems. For example, Dubal et al. (1992) used the multiquadric approximation scheme for the solution of a three-dimensional elliptic partial differential equation describing the time-evolution of interacting black holes and gravitational wave production. Dong and Cheung (2004) utilized Kansa method to solve elastic inclusion problems. Zheng and Li (2008) used Kansa method to investigate acoustic wave propagations. Simonenko et al. (2014) used RBF method to solve elastostatic problems. Pang et al. (2015) applied the Kansa method to the space-fractional advection-dispersion equations. Fallah et al. (2019) used Kansa approach to solve seepage problems. Despite all the advantages of the Kansa method, this method is associated with an unresolved problem that prevents its application to challenging engineering and scientific issues - the problem of choosing optimal value of shape parameter in RBF. For this reason, the problem of choosing the shape parameter was and still is the subject of great scientific debate. Currently, one of the most popular and most established algorithms for finding good value of shape parameter is the Fasshauer's algorithm which was invented by Fasshauer

Olaf Popczyk, MSc.: Department of Computational Mechanics and Engineering, Silesian University of Technology Stanisława Konarskiego 18A, 44-100 Gliwice; PL, Olaf.Popczyk@polsl.pl

Grzegorz Dziatkiewicz, DSc.: Department of Computational Mechanics and Engineering, Silesian University of Technology Stanisława Konarskiego 18A, 44-100 Gliwice; PL, Grzegorz.Dziatkiewicz@polsl.pl 
and Zhang (2007). They adapted leave-one-out cross validation algorithm so that it could be used to find a good value of shape parameter solving PDE problems using the Kansa method together with the multiquadric function.

This article examines the usability of the Fasshauer's algorithm in solving a heat flow problem, and proposes an alternative algorithm based on the Fasshauer's algorithm.

\section{Methods}

As mentioned in introduction, the heat equation is considered in the work:

$$
\gamma \rho u_{t}=\kappa_{x} u_{x}+\kappa_{x x} u,
$$

$\gamma$ is specific heat capacity, $\rho$ is density, and $\kappa$ is thermal conductivity. The solution of Eq. (1) was performed using the Kansa method in combination with the general multiquadric RBF:

$$
\varphi\left(x, x_{j}\right)=\left(\left(x-x_{j}\right)^{2}+\varepsilon^{2}\right)^{p},
$$

$\varepsilon$ is shape parameter. In the Kansa method, it is assumed that the value of an unknown variable at a given point can be expressed as a linear combination of RBFs

$$
u(x)=\sum_{j=1}^{n} \phi_{j}\left(x, x_{j}\right) c_{j} .
$$

Which can be written in matrix form after taking into account all collocation points

$$
\boldsymbol{u}=\boldsymbol{\phi c},
$$

where $c$ is coefficient vector. Consequently

$$
\begin{gathered}
u_{x}=\phi_{x} c \\
u_{x x}=\phi_{x x} c
\end{gathered} \Rightarrow \begin{gathered}
u_{x}=\phi_{x} \phi^{-1} u \\
u_{x x}=\phi_{x x} \phi^{-1} u
\end{gathered}
$$

Substitiution of Eq. (5) and time integration scheme

$$
u_{t} \approx \frac{u_{k+1}-u_{k}}{\Delta t}
$$

to heat Eq. (1) leads after some rearrengements to

$$
\left(\boldsymbol{\gamma} \rho-\Delta t\left(\kappa_{x} \phi_{x}+\kappa \phi_{x x}\right) \phi^{-1}\right) u_{k+1}=(\gamma \rho) u_{k} .
$$

Eq. (7) is fundamental equation solved in the work in order to obtain unsteady variable field $u(x, t)$. Boundary conditions are imposed at each time step by replacing the equations corresponding to the boundary points in the system of Eq. (7) with equations describing the boundary conditions.

In order to investigate the quality of the solutions, two error measures were used:

- PSK-ANAL which is mean percentage error between numerical solution from last time step and analytical solution for steady state,

- PSK-FDM which is mean percentage error between psuedospectral Kansa method solution and finite difference method (FMD) solution evaluated for all time steps.

As part of the article, it is proposed - as mentioned in the introduction - to modify the Fasshauer's algorithm in such a way that instead of looking for the minimum cost function, one is looking for the shape parameter value for which oscillatory behaviour appears in the cost function graph. Visualization of these two values is shown in Fig. 1.

\section{Numerical results}

As part of the numerical study, four simulations were carried out with various numerical setups, they are summarized in Tab. 1. Fig. 2 summarizes the shape parameter values obtained using the Fasshauer's algorithm and its modification as well as the corresponding error measures. 


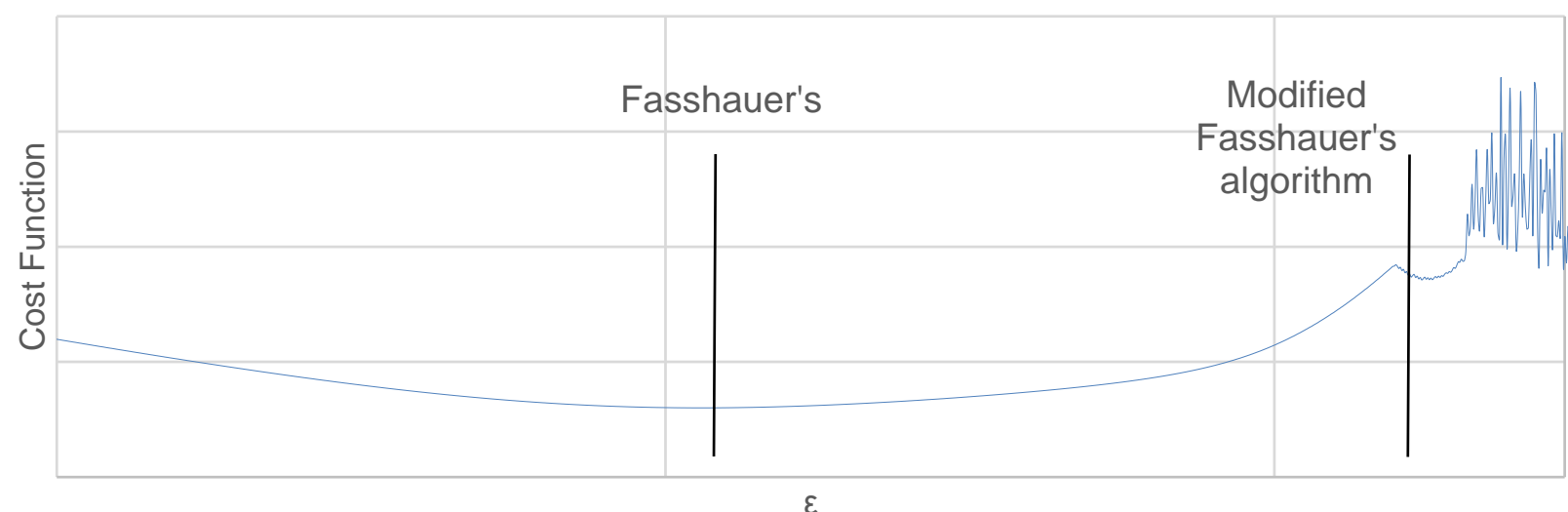

Fig. 1: Sample of graph of the cost function with indicated values of $\varepsilon$ corresponding to Fasshauer's algorithm and modified Fasshauer's algorithm.

Tab. 1: Numerical setups of test cases simulated in the work.

\begin{tabular}{|c|c|c|c|c|}
\hline Test Case & 1 & 2 & 3 & 4 \\
\hline$p$ & 0.5 & 0.4 & 0.4 & -0.5 \\
\hline Thermal parameters distribution & Discontinous & Harmonic & Exponential & Linear \\
\hline Number of collocation points & 240 & 200 & 160 & 120 \\
\hline Boundary condition at left edge & Robin & Neumann & Dirichlet & Neumann \\
\hline Boundary condition at right edge & Robin & Robin & Robin & Dirichlet \\
\hline Time-step size & $0.1 \mathrm{~s}$ & $0.5 \mathrm{~s}$ & $1 \mathrm{~s}$ & $2 \mathrm{~s}$ \\
\hline
\end{tabular}

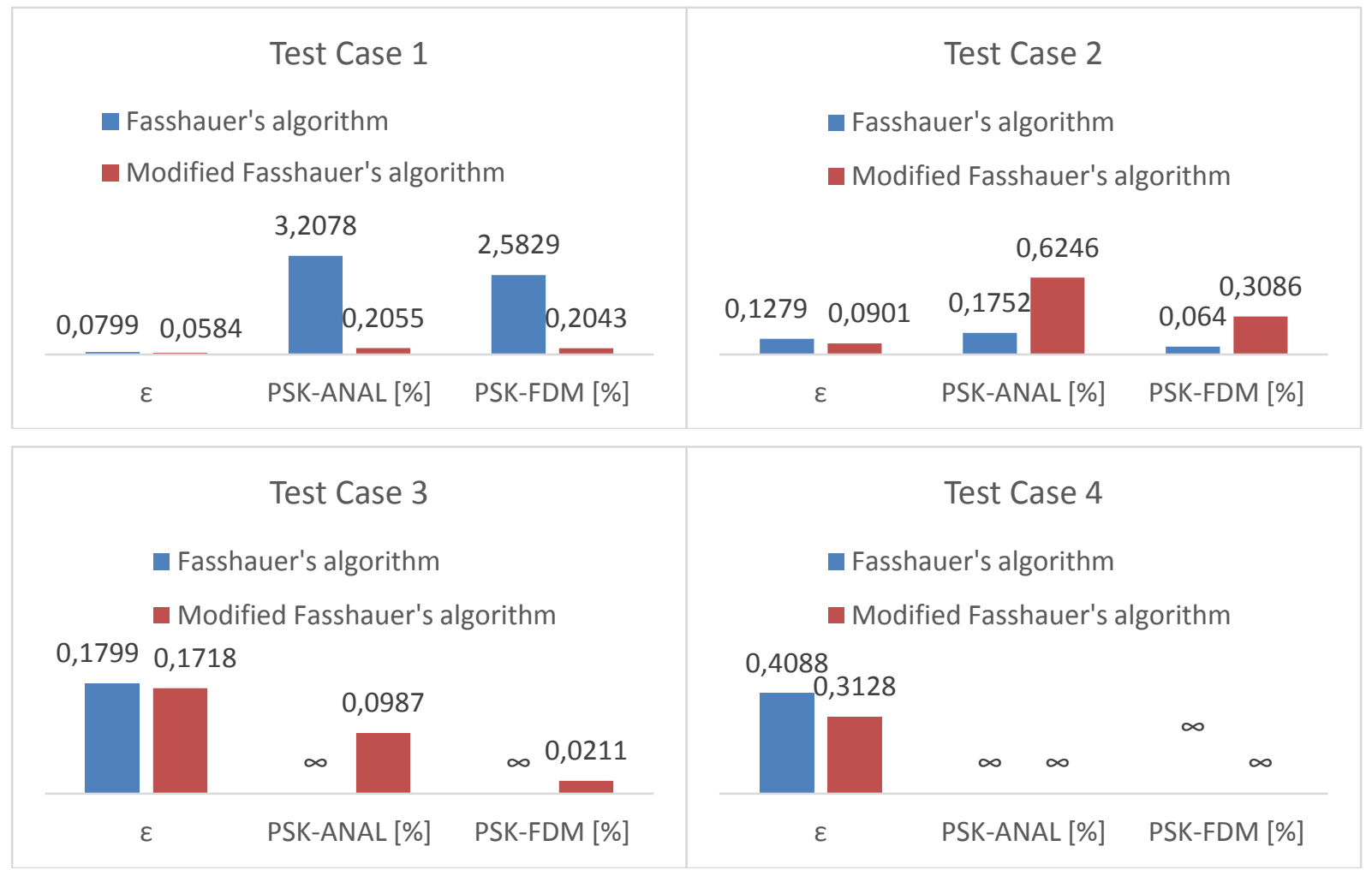

Fig. 2: Comparison of error measures for four considered test cases. $\infty$ indicates that the simulation was unstable due to too high value of shape parameter. 


\section{Conclusions}

The study showed that the Fasshauer algorithm clearly encounters problems in finding a good epsilon value for the problem of unsteady heat flow in a medium with spatially variable thermal parameters. In two of the four analyzed cases (test case 3 and 4), the Fasshauer algorithm gave a shape parameter that led to the ill-conditioned interpolation matrix. In turn, in one of the cases (test case 1) it gave a fairly high error value, over $3.2 \%$. The proposed modification of the Fasshauer algorithm gives clearly better results, only in one case (test case 4) it gave shape parameter which led to ill-conditioned interpolation matrix. In the remaining three cases, the error did not exceed $1 \%$. In summary, for the problem of unsteady heat flow in a medium with spatially variable thermal parameters it is recommended to use the modified Fasshauer's algorithm.

\section{Acknowledgement}

The research is partially financed from financial resources from the statutory subsidy of the Faculty of Mechanical Engineering, Silesian University of Technology in 2020.

\section{References}

Dong, C., Lo, S. and Cheung, Y. (2004) Numerical solution for elastic inclusion problems by domain integral equation with integration by means of radial basis functions. Engineering Analysis with Boundary Elements, 28(6), pp. 623-632.

Dubal, M., Oliveira, S. and Matzner, R. (1992) Solution of elliptic equations in numerical relativity using multiquadrics. Approaches to Numerical Relativity, pp. 265-280.

Fallah, A., Jabbari, E. and Babaee, R. (2019) Development of the Kansa method for solving seepage problems using a new algorithm for the shape parameter optimization. Computers \& Mathematics with Applications, 77(3), pp. 815-829.

Fasshauer, G. E. and Zhang, J. G. (2007) On choosing "optimal” shape parameters for rbf approximation. Numerical Algorithms, 45(1-4), pp. 345-368.

Huang, G., Liu, J., Fu, T., Zhao, Y., Lu, S. and Liu, B. (2018) High-temperature thermal manipulator using thermal metamaterials based on transformation thermodynamics. AIP Advances, 8(8):085120.

Kansa, E. J. (1990) Multiquadrics - a scattered data approximation scheme with applications to computational fluiddynamics I surface approximations and partial derivative estimates. Computers \& Mathematics with applications, 19, 8-9, pp. 127-145.

Kansa, E. J. (1990) Multiquadrics - a scattered data approximation scheme with applications to computational fluiddynamics II solutions to parabolic, hyperbolic and elliptic partial differential equations. Computers \& mathematics with applications, 19, 8-9, pp. 147-161.

Pang, G., Chen, W. and Fu, Z. (2015) Space-fractional advection-dispersion equations by the kansa method. Journal of Computational Physics, 293, pp. 280-296.

Simonenko, S., Bayona, V. and Kindelan, M. (2015) Optimal shape parameter for the solution of elastostatic problems with the rbf method. Journal of Engineering Mathematics, 85(1), pp. 115-129.

Tschikin, M., Biehs, S., Ben-Abdallah, P., Lang, S., Petrov, A. and Eich, M. (2015) Radiative heat flux predictions in hyperbolic metamaterials. Journal of Quantitative Spectroscopy and Radiative Transfer, 158:17-26.

Vemuri. K., Canbazoglu, F. and Bandaru, P. (2014) Guiding conductive heat flux through thermal metamaterials. Applied Physics Letters, 105(19):193904.

Zheng, Z. and Li, W. (2008) Numerical stabilities and boundary conditions in time-domain eulerian simulations of acoustic wave propagations with and without background flow. Applied Mathematics and Computation, 202(1), pp. 146-161. 